\title{
Impact of Baseline LDL-C and Lp(a) Elevation on Coronary Revascularization in Patients with Acute Coronary Syndrome One-Year after First Percutaneous Coronary Intervention
}

\author{
Qarqeen A. Hameed ${ }^{1}$, Lijuan Chen', Mingming Yang1, Jawad Afzali2, Genshan Ma1* \\ ${ }^{1}$ Department of Cardiology, Zhongda Hospital Affiliated to Southeast University, Nanjing, China \\ ${ }^{2}$ Department of Neurology, Zhongda Hospital Affiliated to Southeast University, Nanjing, China \\ Email: *magenshan@hotmail.com
}

How to cite this paper: Hameed, Q.A., Chen, L.J., Yang, M.M., Afzali, J. and Ma, G.S. (2020) Impact of Baseline LDL-C and Lp(a) Elevation on Coronary Revascularization in Patients with Acute Coronary Syndrome One-Year after First Percutaneous Coronary Intervention. World Journal of Cardiovascular Diseases, 10, 102-115. https://doi.org/10.4236/wjcd.2020.102012

Received: January 10, 2020

Accepted: February 18, 2020

Published: February 21, 2020

Copyright $\odot 2020$ by author(s) and Scientific Research Publishing Inc. This work is licensed under the Creative Commons Attribution International License (CC BY 4.0).

http://creativecommons.org/licenses/by/4.0/

\begin{abstract}
Objective: The aim of this study was to investigate the effect of Lipoprotein-a [Lp(a)] on Coronary Revascularizaton (CR) on one year follow up in patients with Acute Coronary Syndrome (ACS) after the first Percutaneous Coronary Intervention (PCI). Method: A retrospective study was designed. A total of 475 patients that underwent their first PCI treatment due to ACS between January 2016 and December 2017 were recruited and followed for one year at the Zhongda Hospital, China. The clinical end point after first PCI was prevalence of Major Adverse Cardiovascular Events (MACE) including nonfatal Myocardial Infarction (MI), cardiovascular death, ischemic stroke and Coronary Revascularization (CR). According to the cut point of $\mathrm{Lp}(\mathrm{a})$, participants were divided into low $\operatorname{Lp}(\mathrm{a})$ subgroup $(\mathrm{Lp}(\mathrm{a})<300 \mathrm{mg} / \mathrm{L})$ and high $\mathrm{Lp}(\mathrm{a})$ subgroup ( $\mathrm{Lp}(\mathrm{a}) \geq 300 \mathrm{mg} / \mathrm{L}$ ). Furthermore, based on baseline Low Density Lipoprotein Cholesterol (LDL-C) level, participants were divided into low LDL-C (LDL-C $<1.8 \mathrm{mmol} / \mathrm{L})$ and high LDL-C (LDL-C $\geq 1.8 \mathrm{mmol} / \mathrm{L})$ subgroups. Results: The number of prevalence of CR was higher with elevated serum Lp(a) in both low LDL-C subgroup and high LDL-C subgroup, and was significantly different in both the low LDL-C subgroup and high LDL-C subgroup ( $\mathrm{p}=0.009$ and $\mathrm{p}=0.006$, respectively). Multivariate Cox-hazard regression analysis for CR showed increase in serum LDL-C and $\mathrm{Lp}(\mathrm{a})$ increased prevalence of CR by 1.514 and 1.002 folds respectively. Furthermore, Kaplan-Meier cumulative survival curves showed that increased prevalence of CR within one year after first PCI in patients with high Lp(a) [ $\log$ rank $\mathrm{p}=$ 0.000]. Conclusion: Baseline increase of serum LDL-C and Lp(a) significantly increases the prevalence of $\mathrm{CR}$ after first PCI within one year. It indicates
\end{abstract}


that after PCI treatment, in patient with serum LDL-C and Lp(a) elevation, treatment with high-dose statin therapy or PCSK9 inhibitors may alleviate the adverse effects imposed by $\mathrm{Lp}(\mathrm{a})$ elevation.

\section{Keywords}

Lipoprotein-a [Lp(a)], Low Density Lipoprotein Cholesterol (LDL-C), Acute Coronary Syndrome (ACS), Coronary Revascularization (CR)

\section{Introduction}

Hyperlipidemia, especially high serum level of low density lipoprotein-cholesterol (LDL-C) has been well documented as an important risk factor of atherosclerosis and its manifestation of atherosclerotic cardiovascular diseases (ASCVD) [1]. Although a significant progress have been made in recent years in the traditional risk factor control and treatment of Acute Coronary Syndrome (ACS). However, there are still a large number of patients with residual risk of cardiovascular events [2]. Hence, some epidemiological studies show that, although target LDL-C level has been achieved by statin therapy, residual cardiovascular risk such as coronary restenosis after Percutaneous Coronary Intervention (PCI) is still very high [3] [4]. It suggests that other risk factors besides LDL-C could also play a contributory role in the progression and recurrence of ACS after PCI, and identifying those potential risk factors would be beneficial for further improving cardiovascular outcome [3] [4].

Recently, some studies show that elevated plasma level of lipoprotein-a $[\mathrm{Lp}(\mathrm{a})]$ and LDL-C is associated with increased risk of Coronary Artery Disease (CAD) [5] [6]. $\mathrm{Lp}(\mathrm{a})$ is a lipoprotein particle with an LDL-like structure linked to apolipoprotein(a) [apo(a)] [6]. So, it has the effect similar to the LDL-C, and also carries atherothrombotic and proinflammatory properties [7] [8]. The combination of all these prothrombotic and atherosclerotic factors could trigger its atherosclerotic property. There is considerable heterogeneity with regards to the plasma level of $\mathrm{Lp}(\mathrm{a})$ among different ethnicities. Its cut-off value of significant risk for the Caucasians was recommended as $500 \mathrm{mg} / \mathrm{L}$, whereas for the Chinese population, it is $300 \mathrm{mg} / \mathrm{L}$ considering far lower than the Caucasians [9] [10].

Currently, PCI is the preferred choice for ACS. The main strategy in the clinical practice after PCI is still focusing on atherogenic lipid profiles other than $\mathrm{Lp}(\mathrm{a})$, especially LDL-C, which is the main treatment target recommended by several guidelines [10] [11]. Although LDL-C has been recognized as one of the major risk factors for ACS, the residual risk for ACS despite intensive statin therapy indicates the important role for $\operatorname{Lp}(\mathrm{a})$ [12]. Therefore, preventing the incidence of coronary atherosclerosis after PCI is of paramount importance. Notably, the pathophysiological process of coronary artery atherosclerosis is featured by endothelial dysfunction, smooth muscle cells proliferation and fibrin 
accumulation, and with respect to $\mathrm{Lp}(\mathrm{a})$ 's unique features such as atherosclerotic and proinflammatory properties, we speculated that $\mathrm{Lp}(\mathrm{a})$ elevation might be the potential risk factor responsible for the increased incidence of $\mathrm{CAD}$ due to coronary restenosis after PCI. A limited number of studies are available on the effect of LDL-C and Lp(a) on ACS patients after PCI treatment in Chinese patients. So, this study was conducted to determine the effect of $\operatorname{Lp}(\mathrm{a})$ elevation on ACS patients within one year after first therapeutic PCI, and also studied whether or not this effect of $\mathrm{Lp}(\mathrm{a})$ is dependent on LDL-C.

\section{Method}

\subsection{Study Design}

A retrospective study was conducted from January 2016 and December 2017 at the Department of Cardiology, Zhongda Hospital, China. All the patients were diagnosed with ACS through thorough angiographic assessment by 2 seasoned cardiologists.

\subsection{Study Population}

In our hospital from January 2016 and December 2017, total 1600 patients have undergone PCI treatments due to Coronary Artery Disease (CAD) and ACS. We have identified and enrolled 475 ACS patients who have undergone first PCI treatment. The study population consisted of patients presented with ACS consisting of Unstable Angina (UA), Non-ST segment Elevation ACS (NSTE-ACS) and ST segment Elevation ACS (STE-ACS) that necessitated therapeutic PCI after thorough angiographic assessment. Of the total enrolled patients, 51 patients were excluded due to in accordance with our exclusion criteria. A total of 424 patients were enrolled in our study (Figure 1). All the patients were prescribed with recommended dose of statin and anti-platelet therapy following PCI and have been followed for one year for any cardiovascular events i.e. Major Adverse Cardiovascular Events (MACE) including, Coronary Revascularization (CR) due to coronary restenosis, hemorrhagic stroke, nonfatal Myocardial Infarction (MI) and cardiovascular death. Baseline demographic and laboratory information (see Table 1), including age, gender, smoking status, lipid profile, hypertension, diabetes mellitus, family history and previous medications, Systolic Blood Pressure (SBP), Diastolic Blood Pressure (DBP) and coronary artery occlusion were obtained retrospectively in all the patients.

The inclusion criteria for current study were 1) patients aged 18 - 80 y/o, 2) the patients undergone first PCI due to any form of CAD i.e. SAP or ACS including Unstable Angina (UA) or STE-ACS or NSTE-ACS. The exclusion criteria was 1) patients with previous PCI, 2) patients with plasma creatinine level of $>132 \mu \mathrm{mol} / \mathrm{L}, 3)$ patients with plasma alanine transaminase level of $>3$ times upper normal limit, 4) patients with unavailability of lipid record, 5) patients with thyrotoxicosis and malignancy or systemic inflammatory disorder.

Accordingly [6], patients after PCI are recommended to achieve target LDL-C 
475 patients with $\mathrm{CAD}$ undergone first $\mathrm{PCI}$ at the department of Cardiology
51 patients excluded in accordance with the exclusion criteria

424 patients enrolled in

the current study

One year follow up

following first PCI

Figure 1. Flow diagram of study population.

Table 1. Comparison of baseline characteristics of low-Lp(a) and high-Lp(a) groups.

\begin{tabular}{|c|c|c|c|c|}
\hline & Variables & $\begin{array}{c}\mathrm{Lp}-\mathrm{a}<300 \mathrm{mg} / \mathrm{L} \\
(\mathrm{n}=256)\end{array}$ & $\begin{array}{c}\mathrm{Lp}-\mathrm{a} \geq 300 \mathrm{mg} / \mathrm{L} \\
(\mathrm{n}=168)\end{array}$ & P-value \\
\hline \multirow[t]{9}{*}{ Demographic } & Age (years) & $67.54 \pm 10.36$ & $65.74 \pm 11.22$ & 0.519 \\
\hline & $\operatorname{Sex}(\%)$ & & & 0.188 \\
\hline & Male (\%) & $164(64.1 \%)$ & $118(70.2 \%)$ & \\
\hline & Female (\%) & $92(35.9 \%)$ & $50(29.8 \%)$ & \\
\hline & Blood Pressure & & & \\
\hline & SBP (mmHg) & $131.52 \pm 19.96$ & $130.92 \pm 19.45$ & 0.990 \\
\hline & $\mathrm{DBP}(\mathrm{mmHg})$ & $85.29 \pm 15.00$ & $84.70 \pm 14.17$ & 0.614 \\
\hline & Smoking (\%) & $79(30.9 \%)$ & $47(28.5 \%)$ & 0.603 \\
\hline & Alcohol (\%) & $74(28.9 \%)$ & $45(27.1 \%)$ & 0.688 \\
\hline Coronary & HTN (\%) & $185(72.3 \%)$ & $120(71.4 \%)$ & 0.851 \\
\hline \multirow[t]{2}{*}{ risk factors } & $\mathrm{DM}(\%)$ & $92(35.9 \%)$ & $57(33.9 \%)$ & 0.672 \\
\hline & Stroke (\%) [previous] & $64(25 \%)$ & $43(25.6 \%)$ & 0.890 \\
\hline \multirow[t]{7}{*}{ Lipid profile } & HDL-C (mmol/L) & $1.06 \pm 0.28$ & $1.08 \pm 0.25$ & 0.748 \\
\hline & LDL-C (mmol/L) & $1.84 \pm 0.72$ & $2.21 \pm 0.88$ & $0.058^{*}$ \\
\hline & $\mathrm{Lp}(\mathrm{a})$ & $140.31 \pm 78.17$ & $617.71 \pm 352.86$ & $0.000^{*}$ \\
\hline & Cholestrol (mmol/L) & $3.52 \pm 0.96$ & $3.71 \pm 1.09$ & 0.104 \\
\hline & Triglyceride $(\mathrm{mmol} / \mathrm{L})$ & $1.55 \pm 1.27$ & $1.49 \pm 1.08$ & 0.201 \\
\hline & Apo-A1 (g/L) & $1.15 \pm 0.26$ & $1.00 \pm 0.28$ & 0.395 \\
\hline & Apo-B (g/L) & $0.59 \pm 0.22$ & $0.67 \pm 0.23$ & 0.829 \\
\hline Laboratory & Glucose (mmol/L) & $6.52 \pm 2.03$ & $6.24 \pm 2.03$ & 0.681 \\
\hline
\end{tabular}




\begin{tabular}{|c|c|c|c|c|}
\hline \multicolumn{5}{|l|}{ Continued } \\
\hline \multirow[t]{2}{*}{ tests } & $\mathrm{S}-\mathrm{Cr}(\mathrm{umol} / \mathrm{L})$ & $86.30 \pm 30.28$ & $92.77 \pm 50.21$ & $0.033^{*}$ \\
\hline & hsCRP (mg/L) & $1.54 \pm 1.03$ & $1.65 \pm 1.15$ & 0.508 \\
\hline Cardiac & CKMB (ng/mL) & $9.61 \pm 22.79$ & $11.70 \pm 20.68$ & 0.493 \\
\hline \multirow[t]{3}{*}{ biomarkers } & $\mathrm{BNP}(\mathrm{pg} / \mathrm{mL})$ & $393.53 \pm 1145$ & $809.13 \pm 1542$ & $0.009^{*}$ \\
\hline & MYO (ng/mL) & $99.20 \pm 165.56$ & $127.42 \pm 156.85$ & 0.318 \\
\hline & $\mathrm{aTnI}(\mathrm{ng} / \mathrm{mL})$ & $0.30 \pm 1.41$ & $0.78 \pm 3.25$ & $0.001^{*}$ \\
\hline Coronary & LM (\%) & $17(6.6 \%)$ & $9(5.4 \%)$ & 0.590 \\
\hline Artery & LAD (\%) & $190(74.2 \%)$ & $128(76.2 \%)$ & 0.647 \\
\hline \multirow[t]{5}{*}{ Occlusion } & LCX (\%) & $101(39.5 \%)$ & $75(44.6 \%)$ & 0.289 \\
\hline & RCA (\%) & $137(53.5 \%)$ & $98(58.3 \%)$ & 0.329 \\
\hline & Single Vessel (\%) & $144(56.2 \%)$ & $86(51.2 \%)$ & 0.306 \\
\hline & Two Vessels (\%) & $52(20.3 \%)$ & $29(17.3 \%)$ & 0.434 \\
\hline & Three Vessels (\%) & $60(23.4 \%)$ & $53(31.5 \%)$ & 0.065 \\
\hline \multirow[t]{6}{*}{ Medications } & Aspirin (\%) & $254(99.2 \%)$ & $166(98.8 \%)$ & \\
\hline & Clopidogrel (\%) & $136(53.1 \%)$ & $92(54.8 \%)$ & \\
\hline & Ticagrelor (\%) & $121(47.3 \%)$ & $76(45.2 \%)$ & \\
\hline & Atorvastatin (\%) & $91(35.5 \%)$ & $61(36.3 \%)$ & \\
\hline & Rosuvastatin (\%) & $165(64.5 \%)$ & $107(63.7 \%)$ & \\
\hline & Metoprolol (\%) & $209(81.6 \%)$ & $136(81 \%)$ & \\
\hline
\end{tabular}

HTN: Hypertension, SBP: Systolic Blood Pressure, DBP: Diastolic Blood Pressure, DM: diabetes mellitus, TC: Total Cholesterol, TG: Triglyceride, S-Cr: Serum Creatinine, hsCRP: high sensitive C-Reactive Protein, LM: left main trunk, HDL-C: High Density Lipoprotein Cholestrole, LDL-C: Low Density Lipoprotein Cholestrol, Apo-A1: Apolipoprotein A1, Apo-B: Apolipoprotein B, CKMB: Creatinine Kinase-MB, BNP: Brain Natriuretic Peptide, MYO: Myoglobin, aTnI: Troponin-I, LAD: Left Anterior Descending Artery, LCX: Left Circumflux Artery, RCA: Rgiht Coronary Artery.

level of less than $1.8 \mathrm{mmol} / \mathrm{L}$; therefore, we used $1.8 \mathrm{mmol} / \mathrm{L}$ of LDL-C as cut-off point to categorize all patients into two subgroups. Plasma level of $\mathrm{Lp}(\mathrm{a})$ lower than $300 \mathrm{mg} / \mathrm{L}$ is considered within the normal range for the Chinese patients, therefore we considered plasma level of $\mathrm{Lp}$ (a) equal to or higher than $300 \mathrm{mg} / \mathrm{L}$ as abnormal [3].

\subsection{Laboratory Measurement}

For variable measurements in the hospital laboratory, blood samples were taken when the patients had been fasting for about 8 hours. Plasma Lp(a) level was analyzed with sandwich Enzyme Linked Immune-sorbent Assays (ELISA kit, Yaji Biosystems, Shanghai, China). All the procedures for variable measurements were performed according to the manual instruction and were evaluated by SYNCHRON LX20 UniCel DxC800 analyzer (Beckman Coulter Inc., USA).

\subsection{Primary End Point}

All the participants have been followed up for the cardiovascular events such as 
MACE. MACE after PCI was prevalence of non-fatal MI, ischemic stroke, cardiovascular death and $\mathrm{CR}$ within the duration of one year. $\mathrm{CR}$ was defined as the angioplasty or PCI within one year after the first PCI. Stroke was defined as the loss of neurological function due to ischemic or hemorrhagic stroke. Cardiovascular death was defined as death due to cardiovascular issues. MI was defined as the prevalence of MI within one year after the first PCI.

\subsection{Statistical Analysis}

Continuous data was presented as mean $\pm \mathrm{SD}$ or median (interquartile range) appropriately and compared by Student's $t$-test when data was normally distributed, otherwise compared by the Wilcoxon rank-sum test. Categorical data was presented as percentage and compared by $\chi^{2}$ test. Cox-hazard regression analysis [Hazard Ratio (HR)] was performed to address predictors for CR. Kaplan-Meier survival estimation analysis was used to address one year survival effect for CR. Statistical analyses were performed by using SPSS software version 20.0 (SPSS, Inc., Chicago, Illinois). A value of $\mathrm{P}<0.05$ was considered significant.

\section{Results}

Baseline Characteristics of CAD patients with $L p-a<300 \mathrm{mg} / L$ subgroup and $L p-a \geq 300 \mathrm{mg} / L$ subgroup One Year after first PCI:

All the enrolled patients were classified into High $\operatorname{Lp}(\mathrm{a})[\mathrm{Lp}(\mathrm{a}) \geq 300 \mathrm{mg} / \mathrm{L}$ ] group $(\mathrm{n}=168)$ and Low $\operatorname{Lp}(\mathrm{a})[\mathrm{Lp}(\mathrm{a})<300 \mathrm{mg} / \mathrm{L}]$ group $(\mathrm{n}=256)$. The baseline characteristics of the enrolled patients of the 2 groups are shown in Table 1. The study population consisted of 282 males (66.5\%) and 142 females (33.5\%), and mean age $66.83 \pm 11$ years. In brief, lipid profile observation has shown that LDL-C and Lp(a) are to be significantly different $(\mathrm{p}<0.05)$ between the two groups. Mean value of HDL-C, Cholestrol, Triglyceride and Apo-B is elevated in patients with high $\mathrm{Lp}(\mathrm{a})$, though they weren't statistically significant between the groups. Observation of lab analysis showed that Serum Creatinine (S-Cr) was significantly different between low and high Lp(a) groups $(\mathrm{p}=0.033)$. The mean value of high sensitive C-Reactive Protein (hsCRP) was higher in high Lp(a) group but was not significant. Meanwhile, the analysis of coronary vessels has shown increased prevalence of stenosis in 3 coronary vessels stenosis, though they weren't significant [53(31.5\%) $(\mathrm{p}=0.065)]$. The stenosis in Right Coronary Artery (RCA), Left Cricumflex Artery (LCX) and Left Anterior Descending (LAD) artery tend to be more in high $\mathrm{Lp}(\mathrm{a})$ group though they were not significant. The values of Brain-type Natriuretic Peptide (BNP) and cardiac Troponin-I (cTnI) were significantly different in high $\operatorname{Lp}(\mathrm{a})$ group $(\mathrm{p}=0.009$ and $\mathrm{p}=$ 0.001 respectively). Meanwhile, the mean value of CKMB and MYO were higher in high $\mathrm{Lp}(\mathrm{a})$ group but they weren't significant. It shows that high level $\mathrm{Lp}(\mathrm{a})$ is associated with necrosis of myocardial fibers.

Cardiovascular Outcomes Comparison in the CAD Patients One Year after first PCI in the Subgroup of $L D L-C<1.8 \mathrm{mmol} / L$ subgroup and $L D L \_C \geq 1.8$ 
$\mathrm{mmol} / \mathrm{L}$ :

Selecting LDL-C level of $1.8 \mathrm{mmol} / \mathrm{L}$ as the cut-off point, 424 patients were divided into two subgroups, low LDL-C (LDL-C $<1.8 \mathrm{mmol} / \mathrm{L})$ and the high LDL-C (LDL-C $\geq 1.8 \mathrm{mmol} / \mathrm{L}$ ) of LDL-C equal or higher than the $1.8 \mathrm{mmol} / \mathrm{L}$. In the subgroup of low LDL-C $(n=211)$ subgroup, there were 147 patients with $\mathrm{Lp}$ (a) level lower than $300 \mathrm{mg} / \mathrm{L}$, while another 64 were with $\mathrm{Lp}$ (a) level higher than $300 \mathrm{mg} / \mathrm{L}$. As shown in Table 2, the number of prevalence of CR was higher as compared to the other adverse events of the total MACE in high Lp(a). In our study prevalence of total MACE and CR was significantly different between low and high $\mathrm{Lp}(\mathrm{a})$ groups $(\mathrm{p}=0.002$ and $\mathrm{p}=0.009$, respectively) in patients with low LDL-C. The prevalence of Stroke was higher in high Lp(a) subgroup and was significantly different $(\mathrm{p}=0.031)$. It shows that elevated level of serum $\mathrm{Lp}(\mathrm{a})$ is a causative factor for adverse events i.e. CR and stroke even after achieving low LDL-C level.

Another 213 patients with LDL-C level equal to or higher than $1.8 \mathrm{mmol} / \mathrm{L}$ were divided into two subgroups according to the cut-off point of $\mathrm{Lp}(\mathrm{a})$. As shown in Table 2, same as in the subgroup with low LDL-C, in the subgroup of patients with high LDL-C level the number of prevalence of CR was higher as compared to the other adverse in total MACE. In this subgroup of patients with high LDL-C level the prevalence of CR and total MACE was significantly different ( $p=0.006$ and $p=0.001$, respectively). Moreover, prevalence of other adverse events of MACE i.e. MI, stroke and Death were higher in high Lp(a) subgroup than the low $\mathrm{Lp}(\mathrm{a})$ subgroup though they weren't significant. So it shows that elevated level of both serum LDL-C and Lp(a) were associated with increased prevalence of MACE especially CR.

Table 2. Cardiovascular outcomes comparison in the subgroup of patients with LDL-C Lower than $1.8 \mathrm{mmol} / \mathrm{L}$ and higher than $1.8 \mathrm{mmol} / \mathrm{L}$ after PCI.

\begin{tabular}{|c|c|c|c|c|}
\hline & & $\begin{array}{c}\mathrm{Lp}-\mathrm{a}<300 \mathrm{mg} / \mathrm{L} \\
(\mathrm{n}=147)\end{array}$ & $\begin{array}{c}\mathrm{Lp}-\mathrm{a} \geq 300 \mathrm{mg} / \mathrm{L} \\
(\mathrm{n}=64)\end{array}$ & $\mathrm{p}$ \\
\hline & MACE & $15(10.2 \%)$ & $17(26.6 \%)$ & $0.002^{*}$ \\
\hline LDL_C & $C R$ & $13(8.8 \%)$ & $14(21.9 \%)$ & $0.009^{*}$ \\
\hline$<1.8 \mathrm{mmol}$ & Stroke & $0(0 \%)$ & $2(3.1 \%)$ & $0.031^{*}$ \\
\hline \multirow[t]{3}{*}{$(\mathrm{n}=211)$} & Death & $1(0.7 \%)$ & $0(0 \%)$ & 0.508 \\
\hline & $M I$ & $1(0.7 \%)$ & $1(1.6 \%)$ & 0.543 \\
\hline & & $\begin{array}{c}\mathrm{Lp}-\mathrm{a}<300 \mathrm{mg} / \mathrm{L} \\
(\mathrm{n}=109)\end{array}$ & $\begin{array}{c}\mathrm{Lp}-\mathrm{a} \geq 300 \mathrm{mg} / \mathrm{L} \\
(\mathrm{n}=104)\end{array}$ & p \\
\hline LDL-C & MACE & $18(16.5 \%)$ & $37(35.6 \%)$ & $0.001^{*}$ \\
\hline$\geq 1.8 \mathrm{mmol} / \mathrm{L}$ & $C R$ & $10(9.2 \%)$ & $24(23.1 \%)$ & $0.006^{*}$ \\
\hline \multirow[t]{3}{*}{$(n=213)$} & Stroke & $3(2.8 \%)$ & $4(3.8 \%)$ & 0.654 \\
\hline & Death & $2(1.8 \%)$ & $4(3.8 \%)$ & 0.375 \\
\hline & $M I$ & $3(2.8 \%)$ & $5(4.8 \%)$ & 0.430 \\
\hline
\end{tabular}

MACE: Major Adverse Cardiovascular Events, CR: Coronary Revascularization, MI: non-fatal Myocardial Infarction, Death: Cardiovascular death, Stroke: Ischemic stroke after PCI. 
Cox Regression Hazards Model for Factors Associated with Coronary Revascularizartion (CR) with CAD after first PCI within One Year.

We performed Cox regression Hazard analysis to analyze the predictors for CR. After performing univariate Cox analysis, significant predictors for CR were Lp(a), total Cholestrol, Triglyceride, Apo-Al, hsCRP, Creatinine and LDL-C, Table 3. Multivariate Cox-analysis showed increase in serum LDL-C and Lp(a) increases the prevalence of CR by 1.514 and 1.002 folds respectively. Multivariate analysis also showed that Total Cholesterol (TC) $[0.651$ (0.507 - 0.835), $\mathrm{p}=$ 0.001], Creatinine (Cr) [0.991 (0.985 - 0.997) $\mathrm{p}=0.003]$ and Triglyceride (TG) [0.775 $(0.635-0.941) \mathrm{p}=0.010]$ were significantly different when combining with all the other factors together.

Kaplan-Meier Survival Estimation Plot for Coronary Revascularization (CR) in Patients with CAD after first PCI within One Year.

We compared the prevalence of CR in CAD patients one year after first PCI between low $\mathrm{Lp}(\mathrm{a})$ group and high $\mathrm{Lp}$ (a) group through Kaplan-Meier cumulative survival analysis as shown in Figure 2. Kaplan-Meier cumulative survival curves for all cause CR for one year showed that the prevalence of CR after the

Table 3. Cox Regression hazards model for univariate and multivariate factors associated with coronary revascularizartion.

\begin{tabular}{|c|c|c|c|c|c|c|c|}
\hline \multicolumn{5}{|c|}{ Univariable } & \multicolumn{3}{|c|}{ Multivariate } \\
\hline Variables & $\mathrm{HR}$ & CI (95\%) & $\mathrm{p}$-value & Variables & $\mathrm{HR}$ & CI (95\%) & p-value \\
\hline Age & 1.001 & $0.994-1.007$ & 0.807 & $\mathrm{Lp}(\mathrm{a})$ & 1.002 & $1.001-1.002$ & 0.000 \\
\hline LDL-C & 1.272 & $1.029-1.571$ & $0.026^{*}$ & LDL-C & 1.514 & $1.125-2.038$ & 0.006 \\
\hline HDL-C & 0.859 & $0.426-1.730$ & 0.670 & $\mathrm{TC}$ & 0.651 & $0.507-0.835$ & 0.001 \\
\hline $\mathrm{TC}$ & 0.819 & $0.676-0.992$ & 0.041 & $\mathrm{Cr}$ & 0.991 & $0.985-0.997$ & 0.003 \\
\hline TG & 0.826 & $0.692-0.986$ & 0.035 & TG & 0.773 & $0.635-0.941$ & 0.010 \\
\hline Apo-A1 & 0.444 & $0.227-0.871$ & 0.018 & & & & \\
\hline Apo-B & 1.534 & $0.780-3.014$ & 0.215 & & & & \\
\hline Gucose & 1.041 & $0.924-1.174$ & 0.505 & & & & \\
\hline hsCRP & 0.611 & $0.442-0.843$ & 0.003 & & & & \\
\hline $\mathrm{Cr}$ & 0.991 & $0.986-0.997$ & 0.005 & & & & \\
\hline SBP & 1.008 & $0.999-1.017$ & 0.076 & & & & \\
\hline DBP & 1.005 & $0.990-1.019$ & 0.534 & & & & \\
\hline HTN & 1.304 & $0.836-2.034$ & 0.241 & & & & \\
\hline $\mathrm{DM}$ & 1.296 & $0.879-1.930$ & 0.203 & & & & \\
\hline Smoking & 0.742 & $0.506-1.090$ & 0.128 & & & & \\
\hline Alcohol & 1.018 & $0.687-1.508$ & 0.929 & & & & \\
\hline $\mathrm{Lp}(\mathrm{a})$ & 1.001 & $1.001-1.002$ & $0.000^{*}$ & & & & \\
\hline
\end{tabular}

HTN: Hypertension, SBP: Systolic Blood Pressure, DBP: Diastolic Blood Pressure, DM: diabetes mellitus, TC: Total Cholesterol, TG: Triglyceride, S-Cr: Serum Creatinine, hsCRP: high sensitive C-Reactive Protein, HDL-C: High Density Lipoprotein Cholestrole, LDL-C: Low Density Lipoprotein Cholestrol, Apo-A1: Apolipoprotein A1, Apo-B: Apolipoprotein B. 


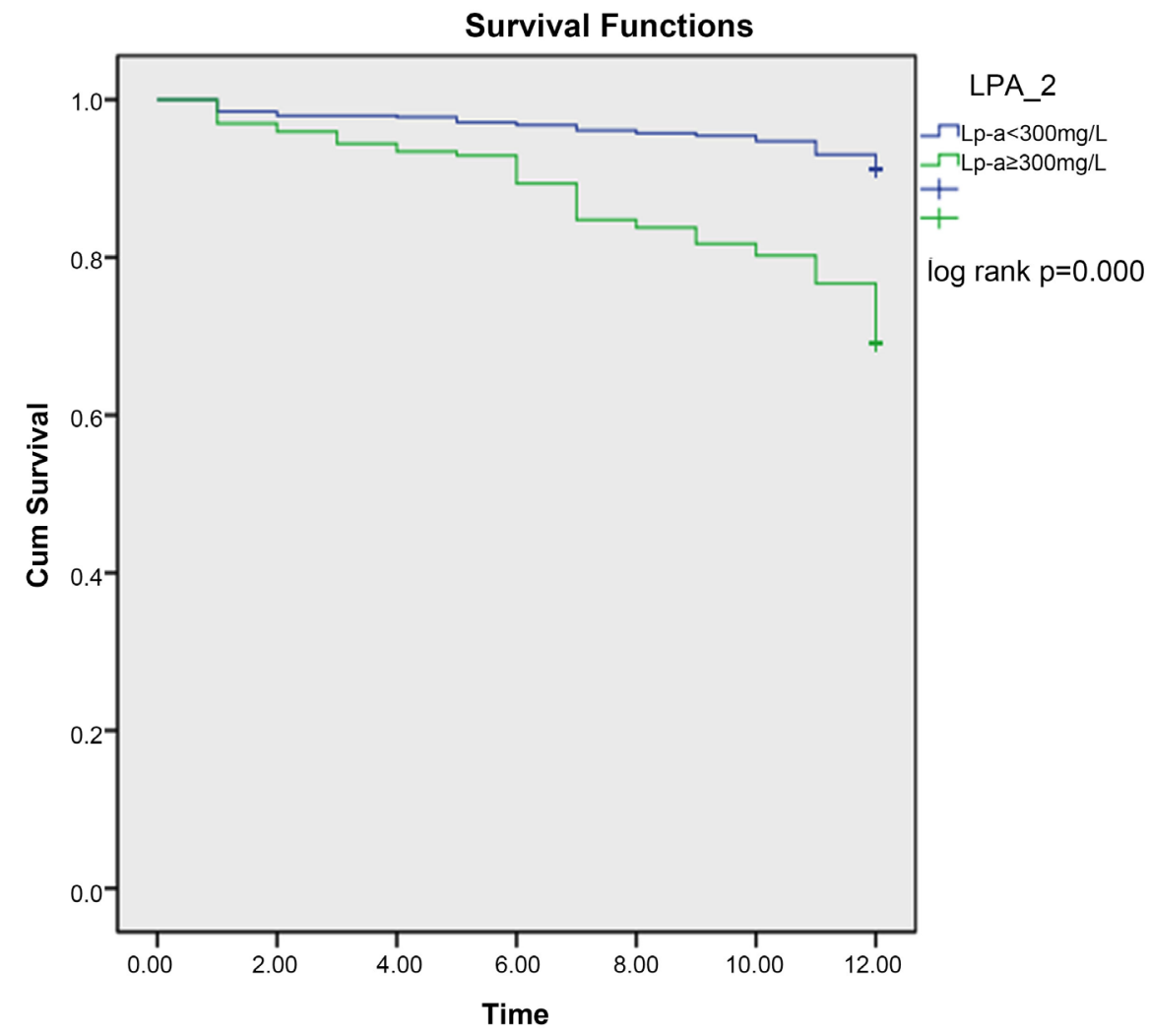

Figure 2. Kaplan-meier survival estimation plot of coronary revascularization (CR) in patients with CAD after first PCI within one year.

first PCI was significantly higher in the group with high $\operatorname{Lp}(a)$ than the low $\operatorname{Lp}(a)$ group $(\log \operatorname{rank} \mathrm{p}=0.000)$.

\section{Discussion}

Our study showed that the number of prevalence of stent-restenosis and CR was higher as compared to the other adverse events of the total MACE in high Lp(a) in patients with low LDL-C subgroup. The increase in serum LDL-C and Lp(a) were associated with increase in prevalence of total MACE and CR. The prevalence of CR after the first PCI was significantly higher in the group with high Lp(a) subgroup than low $\mathrm{Lp}$ (a) subgroup. Our study also showed that LDL-C and $\mathrm{Lp}(\mathrm{a})$ increases prevalence of CR by 1.514 and 1.002 folds respectively. This association was significant even after adjustment for other independent variables. After the treatment with PCI all the patients were well followed up and were prescribed with recommended medications such as antiplatelet and statin therapies.

At present, PCI is largely applied in patients with significant coronary artery stenosis, and this reperfusion approach has overwhelmingly enhanced cardiovascular outcomes during last two decades. However, the accumulation of evidence from retrospective and perspective studies show that, despite the patients treated with PCI and strictly adhering to recommended guidelines, a significant 
number of patients still have a high residue of cardiovascular risk, especially the revascularization of the target vessel requiring atherosclerosis. With regard to these findings, many strategies to reduce residual cardiovascular risk have been introduced, and a more aggressive LDL-C reduction treatment is considered as one of the most attractive and potential strategy.

Recently LIPID [13] study has shown the relationship between serum Lp(a) and further CVD events in patients with CAD on statin therapy. Formerly, observation of patients with CAD treated with intensive statin therapy in PROVE IT-TIMI [14], IDEAL [4] and TNT [15] clinical trials have shown, during their follow-ups they have found increased frequency of CR even with intensive statin treatment. In addition, the IDEAL and TNT trials have shown residual CVD risk during their 5 years follow up (12.0\% and 8.7\%, respectively). The results of these important clinical trials have repeatedly shown that despite aggressive reduction of LDL-C, significant cardiovascular residual risks remain in the patient and that LDL-C alone is not sufficient to reduce cardiovascular risk. Although $\mathrm{Lp}$ (a) was an attractive topic in clinical research, until recently it was widely accepted and considered a unique predictor of CAD risk. Evidence from genetic study show that $\operatorname{Lp}(\mathrm{a})$ is more of a causal risk factor for cardiovascular disease than just a marker [16]. There were considerable studies that have been previously reported about the possible role of $\mathrm{Lp}(\mathrm{a})$ in the development of CAD [17] [18] [19]. Lp(a) could be a risk factor for plaque destabilization and thrombosis and not the severity of coronary heart disease, and associated with myocardial lesions in patients with unstable angina [17]. High concentrations of serum $\mathrm{Lp}$ (a) could predict ischaemic heart disease (IHD) and myocardial infarction (MI) in the general public, and the predictive capability was not exaggerated by normal food intake, but was minimally elevated to a level high CRP [18]. A study by Juan Chen et al. demonstrated that $\mathrm{Lp}(\mathrm{a})$ is an independent predictor for the severity of coronary heart disease (CHD) in type 2 diabetes patients (T2DM) [19]. It has been shown that $\mathrm{Lp}(\mathrm{a})$ has shown that the severity of coronary atherosclerosis is predicted independently of conventional CV risk factors. [20]. In a study, Cho et al. demonstrated that high serum levels of $L p(a)$ were significantly related to long-term side effects in Acute Myocardial Infarction (AMI) patients, particularly in patients with a high killip class. Proatherogenic and prothrombotic effects of $\mathrm{Lp}(\mathrm{a})$ might be associated with adverse CV events throughout follow-up.

We therefore speculate that the possible pathophysiological mechanism for CAD might be due to the atherothrombotic and pro-inflammatory properties of Lp(a) [7] [8]. There are some possible mechanisms behind atherosclerosis and restenosis after PCI. 1) Lp(a) inhibits the activation of the transforming growth factor and causes growth of atherosclerotic arterial lesions via increased vascular smooth muscle cells and the migration of muscle cells smooth endothelial cells [21] [22]. 2) Lp(a) inhibits plasminogen binding site on the surface of endothelial cells and reduces the activity of the tissue-type Plasminogen Activator (t-PA). 
3) $\mathrm{Lp}$ (a) directly increases the activity of plasminogen activator inhibitors in human endothelial cells and promotes atherorombosis [23]. Lp(a) was involved in the activation of endothelial uptake, oxidative alteration and cell formation. Additionally, $\mathrm{Lp}(\mathrm{a})$ is also associated with 1) thrombus formation due to vascular lesion, 2) elastic recoil, 3) migration of smooth muscle cells in Intima and 4) vascular remodelling. A study by Minz et al. [24] used intravascular ultrasound to study CAD patients for coronary restenosis through Percutaneous angiography without stent treatment and reported that $73 \%$ of the mechanism underlying a decrease in lumen diameter is because of remodelling and $27 \%$ is due to an increase in plaque and intima. On the other hand, another study by Hoffmann et al. [25] demonstrated that in-stent restenosis results from neo-intimal tissue proliferation, which has been accredited due to $\mathrm{Lp}(\mathrm{a})$ in vitro [8]. In the present study, Lp(a) was significantly associated with CR which is in parallel with the previous studies. Recently a meta-analysis on statin therapy following PCI show beneficial role of high intensity loading dose of statin therapy was more beneficial to patients with ACS in Chinese patients and was suitable for clinical application [26]. In addition, a study by Zhi Liu et al. [27] demonstrated that high-intensity atorvastatin therapy over the duration of 1 year after PCI was superior in ACS patients in decreasing MACE. Moreover, novel drugs, including PCSK9 inhibitors, as well as antisense oligonucleotide for Apo-A, have exhibited efficacy in decreasing Lp(a) substantially [28] [29].

Several limitations of this retrospective study should be given consideration. It's a single-center retrospective study, enrolling data of 2 years of patients and other confounding factors might have influenced the outcomes independently of adjustments in the statistical analysis. Thus an observational study with larger population is required for further proper analysis. In addition we had no proper knowledge about the patients' compliance with the prescribed medications.

\section{Conclusion}

In conclusion, this study revealed that in patients with first PCI, Lp(a) elevation is positively associated with coronary restenosis and CAD within one year which requires revascularization and the prevalence of $\mathrm{CR}$ is significantly higher in elevated level of serum LDL-C and Lp(a). It indicates that after PCI treatment, in patient with serum LDL-C and Lp(a) elevation, treatment with high-dose statin therapy or PCSK9 inhibitors may alleviate the adverse effects imposed by Lp(a) elevation.

\section{Acknowledgements}

The authors are thankful to Yu Xiaojin and Min Jie for their assistance in statistical analysis.

\section{Funding}

The authors received no financial support for this study. 


\section{Author's Contribution}

All the authors read and approved this study for publication.

\section{Conflicts of Interest}

The authors declared no potential conflicts of interest with respect to the research, authorship, and/or publication of this article.

\section{References}

[1] Reiner, Z., et al. (2011) ESC/EAS Guidelines for the Management of Dyslipidaemias: The Task Force for the Management of Dyslipidaemias of the European Society of Cardiology (ESC) and the European Atherosclerosis Society (EAS). European Heart Journal, 32, 1769-1818. https://doi.org/10.1093/eurheartj/ehr158

[2] Laschkolnig, A., et al. (2014) Lipoprotein (a) Concentrations, Apolipoprotein (a) Phenotypes, and Peripheral Arterial Disease in Three Independent Cohorts. Cardiovascular Research, 103, 28-36. https://doi.org/10.1093/cvr/cvu107

[3] Cannon, C.P., et al. (2004) Intensive versus Moderate Lipid Lowering with Statins after Acute Coronary Syndromes. The New England Journal of Medicine, 350, 1495-1504. https://doi.org/10.1056/NEJMoa040583

[4] LaRosa, J.C., et al. (2005) Intensive Lipid Lowering with Atorvastatin in Patients with Stable Coronary Disease. The New England Journal of Medicine, 352, 1425-1435. https://doi.org/10.1056/NEJMoa050461

[5] Stone, N.J., et al. (2014) 2013 ACC/AHA Guideline on the Treatment of Blood Cholesterol to Reduce Atherosclerotic Cardiovascular Risk in Adults. Journal of the American College of Cardiology, 63, 2889-2934. https://doi.org/10.1016/j.jacc.2013.11.002

[6] Nordestgaard, B.G., et al. (2010) Lipoprotein (a) as a Cardiovascular Risk Factor: Current Status. European Heart Journal, 31, 2844-2853.

https://doi.org/10.1093/eurheartj/ehq386

[7] Imhof, A., et al. (2003) Plasma Lipoprotein Lp (a), Markers of Haemostasis and Inflammation, and Risk and Severity of Coronary Heart Disease. The European Journal of Cardiovascular Prevention \& Rehabilitation, 10, 362-370. https://doi.org/10.1097/01.hjr.0000087080.83314.be

[8] Grainger, D., et al. (1993) Proliferation of Human Smooth Muscle Cells Promoted by Lipoprotein (a). Science, 260, 1655-1658.

https://doi.org/10.1126/science.8503012

[9] Joint Committee for Developing Chinese Guidelines on Prevention and Treatment of Dyslipidemia in Adults (2007) Chinese Guidelines on Prevention and Treatment of Dyslipidemia in Adults. Chinese Journal of Cardiovascular Diseases, 35, 390-419.

[10] Catapano, A.L., et al. (2016) 2016 ESC/EAS Guidelines for the Management of Dyslipidaemias. European Heart Journal, 37, 2999-3058.

https://doi.org/10.1093/eurheartj/ehw272

[11] Bjornsson, E., et al. (2015) Common Sequence Variants Associated With Coronary Artery Disease Correlate with the Extent of Coronary Atherosclerosis. Arteriosclerosis, Thrombosis, and Vascular Biology, 35, 1526-1531. https://doi.org/10.1161/ATVBAHA.114.304985

[12] Khera, A.V., et al. (2014) Lipoprotein (a) Concentrations, Rosuvastatin Therapy, and Residual Vascular Risk. Circulation, 129, 635-642. 
https://doi.org/10.1161/CIRCULATIONAHA.113.004406

[13] Nestel, P.J., et al. (2013) Plasma Lipoprotein (a) Concentration Predicts Future Coronary and Cardiovascular Events in Patients with Stable Coronary Heart Disease. Arteriosclerosis, Thrombosis, and Vascular Biology, 33, 2902-2908. https://doi.org/10.1161/ATVBAHA.113.302479

[14] Virani, S.S., et al. (2012) Associations Between Lipoprotein (a) Levels and Cardiovascular Outcomes in Black and White Subjects. Circulation, 125, 241-249. https://doi.org/10.1161/CIRCULATIONAHA.111.045120

[15] Pedersen, T.R., et al. (2005) High-Dose Atorvastatin vs Usual-Dose Simvastatin for Secondary Prevention after Myocardial Infarction: The IDEAL Study: A Randomized Controlled Trial. The Journal of the American Medical Association, 294, 2437-2445. https://doi.org/10.1001/jama.294.19.2437

[16] Boffa, M.B. and Koschinsky, M.L. (2013) Screening for and Management of Elevated Lp (a). Current Cardiology Reports, 15, 417. https://doi.org/10.1007/s11886-013-0417-8

[17] Yazici, M., et al. (2006) The Importance of Lipoprotein (a) in Pathogenesis of the High Risk Unstable Angina. Anadolu Kardiyoloji Dergisi, 6, 13-17.

[18] Langsted, A., Kamstrup, P.R. and Nordestgaard, B.G. (2014) Lipoprotein (a): Fasting and Nonfasting Levels, Inflammation, and Cardiovascular Risk. Atherosclerosis, 234, 95-101. https://doi.org/10.1016/j.atherosclerosis.2014.01.049

[19] Chen, J., et al. (2015) Role of Lipoprotein (a) in Predicting the Severity of New on-Set Coronary Artery Disease in Type 2 Diabetics: A Gensini Score Evaluation. Diabetes and Vascular Disease Research, 12, 258-264. https://doi.org/10.1177/1479164115579004

[20] Lima, L.M., et al. (2006) Increased Serum Levels of Lipoprotein (a) Correlated with the Severity of Coronary Artery Disease in Patients Submitted to Angiography. Arquivos Brasileiros de Cardiologia, 87, 260-266. https://doi.org/10.1590/S0066-782X2006001600006

[21] Kojima, S., Harpel, P.C. and Rifkin, D.B. (1991) Lipoprotein (a) Inhibits the Generation of Transforming Growth Factor Beta: An Endogenous Inhibitor of Smooth Muscle Cell Migration. Journal of Cell Biology, 113, 1439-1445. https://doi.org/10.1083/jcb.113.6.1439

[22] Lawn, R.M., et al. (1996) Feedback Mechanism of Focal Vascular Lesion Formation in Transgenic Apolipoprotein (a) Mice. The Journal of Biological Chemistry, 271, 31367-31371. https://doi.org/10.1074/jbc.271.49.31367

[23] Etingin, O.R., Hajjar, D.P., Hajjar, K.A., Harpel, P.C. and Nachman, R.L. (1991) Lipoprotein (a) Regulates Plasminogen Activator Inhibitor-1 Expression in Endothelial Cells. A Potential Mechanism in Thrombogenesis. The Journal of Biological Chemistry, 266, 2459-2465.

[24] Mintz, G.S., et al. (1996) Arterial Remodeling after Coronary Angioplasty: A Serial Intravascular Ultrasound Study. Circulation, 94, 35-43. https://doi.org/10.1161/01.CIR.94.1.35

[25] Hoffmann, R., et al. (1996) Patterns and Mechanisms of in-Stent Restenosis: A Serial Intravascular Ultrasound Study. Circulation, 94, 1247-1254. https://doi.org/10.1161/01.CIR.94.6.1247

[26] Ye, Z., et al. (2017) Effect of High-Dose Rosuvastatin Loading before Percutaneous Coronary Intervention in Chinese Patients with Acute Coronary Syndrome: A Systematic Review and Meta-Analysis. PLoS ONE, 12, e0171682. 
https://doi.org/10.1371/journal.pone.0171682

[27] Liu, Z., et al. (2016) Efficacy of High-Intensity Atorvastatin for Asian Patients Undergoing Percutaneous Coronary Intervention. Annals of Pharmacotherapy, 50, 725-733. https://doi.org/10.1177/1060028016654722

[28] Li, N., Li, Q., Tian, X.Q., Qian, H.Y. and Yang, Y.J. (2014) Mipomersen Is a Promising Therapy in the Management of Hypercholesterolemia: A Meta-Analysis of Randomized Controlled Trials. American Journal of Cardiovascular Drugs, 14, 367-376. https://doi.org/10.1007/s40256-014-0077-0

[29] Du, H., et al. (2019) Proprotein Convertase Subtilisin/Kexin 9 Inhibitors in Reducing Cardiovascular Outcomes: A Systematic Review and Meta-Analysis. Heart, 105, 1149-1159. 